\title{
$\begin{array}{ll}\text { Research Square } & \text { Preprints are preliminary reports that have not undergone peer review. } \\ \text { They should not be considered conclusive, used to inform clinical practice, }\end{array}$
}

\section{Deletions of the Plasmodium falciparum histidine-rich protein $2 / 3$ genes are common in field isolates from north-eastern Tanzania}

Robert D. Kaaya ( $\square$ robertkaaya@yahoo.com )

Kilimanjaro Christian Medical University College

Reginald A. Kavishe

Kilimanjaro Christian Medical University College

Filemon F. Tenu

Pan-African Malaria Vector Research Consortium

Johnson J. Matowo

Kilimanjaro Christian Medical University College

Franklin W. Mosha

Kilimanjaro Christian Medical University College

Chris Drakeley

London School of Hygiene \& Tropical Medicine

Colin J. Sutherland

London School of Hygiene \& Tropical Medicine

Khalid B. Beshir

London School of Hygiene \& Tropical Medicine

\section{Research Article}

Keywords: Plasmodium falciparum, malaria, pfhrp2, pfhrp3, rapid diagnostic tests

Posted Date: November 9th, 2021

DOI: https://doi.org/10.21203/rs.3.rs-1064772/v1

License: (c) (i) This work is licensed under a Creative Commons Attribution 4.0 International License. Read Full License

Version of Record: A version of this preprint was published at Scientific Reports on April 6th, 2022. See the published version at https://doi.org/10.1038/s41598-022-09878-3. 


\section{Abstract}

Plasmodium falciparum parasites lacking histidine-rich protein 2 and 3 (pfhrp2/3) genes have been reported in several parts of the world. These deletions are known to compromise the effectiveness of HRP2-based malaria rapid diagnostic tests (HRP2RDT). The National Malaria Control Programme (NMCP) in Tanzania adopted HRP2-RDTs as a routine tool for malaria diagnosis in 2009 replacing microscopy in many Health facilities. We investigated pfhrp2/3 deletions in 122 samples from two areas with diverse malaria transmission intensities in Northeastern Tanzania. Pfhrp2 deletion was confirmed in $1.6 \%$ of samples while pfhrp3 deletion was confirmed in $50 \%$ of samples. We did not find parasites with both pfhrp 2 and pfhrp3 deletions among our samples. Results from this study highlight the need for systematic surveillance of $p f h r p 2 / 3$ deletions in Tanzania to understand their prevalence and determine their impact on the performance of mRDT.

\section{Introduction}

Malaria continues to be a health problem in Sub-Saharan Africa (SSA), where the 10 countries with the highest infection rates and deaths in the world are found ${ }^{1}$. A recent resurgence of the disease is evident in areas where a significant decline in malaria cases was previously observed and this inevitably calls for scrutiny of the malaria control interventions currently in use, ${ }^{1}$ which comprise a number of strategies ${ }^{1,2}$. Artemisinin-based combination therapy (ACT) is the approved regimen for malaria treatment ${ }^{3}$. The World Health Organization (WHO) recommends for parasitological confirmation to be carried out before this treatment is provided ${ }^{4}$. Microscopy, although still regarded as the gold standard in malaria diagnosis, faces several operational challenges ${ }^{5,6}$. In the resource-limited settings of SSA, shortage of personnel with expertise, and long turn-around time of results from the laboratory have been identified as obstacles to the effective microscopic diagnosis of malaria, particularly in rural settings ${ }^{7,8}$.

Rapid diagnostic tests have proven to be reliable and sensitive enough to replace microscopy as a routine technique for malaria diagnosis in symptomatic patients ${ }^{9}$. The National Malaria Control Program (NMCP) in Tanzania rolled out malaria Rapid Diagnostic Tests (mRDT) in 2009, achieving diagnostic coverage of around $90 \%$ in public and private health facilities in 2014 , with microscopy being used in the remaining $10 \%$ of facilities in the country ${ }^{10}$. Rapid diagnostic tests have different detection thresholds, but sensitivity and specificity decrease when parasitaemia is below 200 parasites/ul ${ }^{11,12}$. Given the high detection threshold, mRDT might miss malaria parasites that are at low densities.

The majority of mRDTs distributed in the SSA are for Plasmodium falciparum detection ${ }^{13}$, mostly utilizing the histidine-rich protein (HRP2), as the antigenic marker. HRP2 is a 60-105 kD water-soluble protein secreted by $P$. falciparum trophozoites (asexual stage), encoded by the sub-telomeric pfhrp2 locus on chromosome $8^{14,15}$. HRP2 is abundantly secreted and easily detected in the peripheral blood circulation of the host even when the parasite has sequestered in the microvasculature of the organs $^{16,17}$, making the protein an important diagnostic target. Over $80 \%$ of mRDTs manufactured worldwide are sold or distributed in SSA. Tanzania Medicines and Medical Devices Authority (TMDA) approved five brands of mRDTs to be used in Tanzania ${ }^{18}$. Accredited Drug Dispensing Outlets (ADDOs) sells an average of 40 RDT units every week, most of them are HRP2based and there are more than 6000 outlets in Tanzania mainland ${ }^{19,20}$.

Studies in the last decade have reported the presence of $P$. falciparum parasites lacking both the loci encoding HRP2 and its isoform HRP3 (encoded by pfhrp3), which has sufficient similarity to HRP2 and is recognised by the monoclonal antibodies used on the RDT test strips. P. falciparum

parasites lacking the locus are thus not detected by HRP2-RDT. In the Amazon region of South America, these deletions have a reported prevalence of $28.6 \%$, leading to recommendations to immediately stop using HRP2RDTs ${ }^{21}$. Evidence from Asian countries also suggests the presence of $P$. falciparum lacking pfhrp2, including in India with a prevalence of $4 \% 22$ and ChinaMaynmar border with a prevalence of $5 \%{ }^{23}$. Across Africa, Eritrea reported the highest prevalence of pfhrp2 and pfhrp3 deletions of $80.8 \%$ and $92.3 \%$, respectively, which prompted a switch to non-HRP2-RDTs ${ }^{24,25}$. Studies elsewhere in East Africa also showed 
evidence of low proportions of pfhrp2/3 gene deletions from field isolates ${ }^{26-29}$. Mention here deletions reported in Tanzania and hence prompted this study.

As Tanzania embarks on a malaria pre-elimination strategy, RDTs will play a crucial role in case detection, and assessment of their performance is vital. This study set out to assess the performance of HRP2-RDTs in two areas in Tanzania with different malaria transmission intensities.

\section{Results}

\section{Malaria parasite detection}

A total of 998 blood samples, 472 from Moshi and 526 from Handeni sites were collected and investigated for pfhrp2/3 deletions using LDH/HRP2-RDT, microscopy and nested PCR. In Moshi site, only 1 sample (0.2\%) was positive by mRDT while in the Handeni site, 203 (38.6\%) samples were positive. Analysis by nested PCR revealed that 135 (13.5\%) samples were positive, of which 19 (4\%) positive samples were from Moshi and 116 (22\%) positives were from Handeni as shown

in Table 1.

Table 1

Malaria prevalence in the Handeni and Moshi district

\begin{tabular}{|c|c|c|c|c|c|c|c|c|c|c|c|c|}
\hline \multirow[b]{3}{*}{ Lower } & \multicolumn{4}{|c|}{ Handeni } & \multicolumn{4}{|l|}{ Moshi } & \multicolumn{4}{|c|}{ Both Sites } \\
\hline & $\mathbf{N}$ & $\%$ & \multicolumn{2}{|c|}{ 95\% C.I. } & $\mathbf{N}$ & $\%$ & \multicolumn{2}{|c|}{ 95\% C.I. } & $\mathbf{N}$ & $\%$ & \multicolumn{2}{|c|}{ 95\% C.I. } \\
\hline & Upper & & Lower & Upper & Lower & Upper & & & & & & \\
\hline $\begin{array}{l}\text { mRTD } \\
\text { positive }\end{array}$ & 203 & 38.59 & 35.42 & 43.88 & 1 & 0.21 & 0.03 & 1.49 & 204 & 20.44 & 18.29 & 23.36 \\
\hline $\begin{array}{l}\text { Microscope } \\
\text { positive }\end{array}$ & 89 & 16.95 & 13.95 & 20.37 & 6 & 1.27 & 0.57 & 2.80 & 95 & 9.52 & 7.85 & 11.50 \\
\hline $\begin{array}{l}\text { PCR } \\
\text { positive }\end{array}$ & 116 & 22.05 & 18.71 & 25.80 & 19 & 4.03 & 2.58 & 6.23 & 135 & 13.53 & 11.54 & 15.80 \\
\hline
\end{tabular}

\section{Confirming pfhrp2/3 deletion}

A total of 122 samples that were positive for $P$. falciparum species-specific PCR or microscopy were analysed for $p$ fhrp2 and pfhrp3 deletions. Of the 122 samples, four samples were pfhrp2 PCR negative, and two of the four samples (1.6\%) had relative parasitaemia of $\geq 5 \mathrm{p} / \mu$ land were considered true pfhrp2 deletion. The two samples with the confirmed pfhrp2 deletion had parasiteamia of $144 \mathrm{p} / \mu \mathrm{l}$ and $440 \mathrm{p} / \mu \mathrm{l}$. Pfhrp3 analysis showed that $52 \%(63 / 122)$ of the samples were negative, and further analysis on parasitaemia revealed that the majority $(50 \%, n=61)$ had a relative parasite density of $\geq 5 / \mu l$ (Figure 1$)$. The parasitaemia of the pfhrp3-deleted samples ranges between $12 \mathrm{p} / \mu \mathrm{l}$ and $14696 \mathrm{p} / \mu \mathrm{l}$, with a mean parasite density of $1473 \mathrm{p} / \mu \mathrm{l}$.

\section{Effect of pfhrp2/3 gene deletion on RDT performance}

Of the 122 samples screened for pfhrp2/3 deletion, discordance analysis showed that four samples but only two samples with pfhrp2 confirmed deletion were RDT positive, while only 2 out of 63 samples with pfhrp3 deletion were RDT negative (Table 2). Overall, there were 32 samples with RDT negative results but pfhrp2/3 positive had a mean parasitaemia of $14 \mathrm{p} / \mu \mathrm{l}$ which ranged between $1 \mathrm{p} / \mu \mathrm{l}$ and $142 \mathrm{p} / \mu \mathrm{l}$. 
Table 2

Discordance between microscopy, nPCR, RDT and pfhrp2/3 PCR

results

\begin{tabular}{|lll|}
\hline N=122 & $\begin{array}{l}\text { nPCR+ or Micro+ \&RDT - } \\
\text { n (\%) }\end{array}$ & $\begin{array}{l}\text { nPCR+ or Micro+\&RDT+ } \\
\mathbf{n}(\%)\end{array}$ \\
\hline Pfhrp2 - & $0(0 \%)$ & $4(3 \%)$ \\
\hline Pfhrp2 + & $16(13 \%)$ & $102(84 \%)$ \\
\hline Pfhrp3 - & $2(1.6 \%)$ & $61(50 \%)$ \\
\hline Pfhrp3 + & $14(11.4 \%)$ & $45(37 \%)$ \\
\hline
\end{tabular}

Impact of parasitaemia on pfhrp2/3 gene detection

In comparison, parasitaemia was $1 \mathrm{p} / \mu \mathrm{l}$ in the pfhrp2 positive and $2 \mathrm{p} / \mu \mathrm{l}$ in the pfhrp2-deleted samples (Figure 2). On the other hand, pfhrp3 positive and pfhrp3-deleted samples had parasitaemia of $1 \mathrm{p} / \mu \mathrm{l}$ and $12 \mathrm{p} / \mu \mathrm{l}$ respectively (Figure 3).

\section{Discussion}

In this study, we report evidence of pfhrp2/3 deletions in north-eastern Tanzania. We confirmed the presence of two samples with pfhrp2 deletion, though they did not cause RDT negative result. This finding is also consistent with previous studies on samples from Tanzania and Yemen, where they found pfhrp2 deletion on an mRDT positive sample ${ }^{30,31}$. This anomaly might be due to false-positive results on mRDT caused by cross-reaction with circulating proteins such as rheumatoid factor ${ }^{32}$ or the positive RDT signal may have arisen from the previous infection with pfhrp2-positive samples ${ }^{33}$. Our results also show a high proportion of pfhrp3 deletion compared to pfhrp2. This finding is interesting, given pfhrp3 deletion is suggested to be more common in low transmission season with minimal chance of polyclonal infection. Reports from Central and Southern America, where malaria transmission is low, showed similar observations, whereby up to $70 \%$ of the tested samples had a deletion on pfhrp3 region 21,34,35. Double deletions (pfhrp2+pfhrp3) were not observed in this study.

Guidelines on pfhrp2/3 deletions rely solely on discordant results between microscopy and mRDT or a dual-antigen HRP2-RDT as an algorithm for suspecting the deletions ${ }^{36}$. All the microscopy positive samples were screened in this analysis, irrespective of symptoms, even if they had positive mRDT results. Studies have shown persistence of HRP2 in the plasma up to 28 days after treatment particularly in high parasitaemia infections ${ }^{33}$, in that case, a person can have a new infection with $P$. falciparum parasites lacking pfhrp2/3 genes but have a circulating HRP2 from the previous infection commonly observed in areas with high malaria transmission. In the absence of the pfhrp2 locus, circulating HRP3 protein alone can give a positive HRP-RDT signal when parasitaemia is more than 1000 parasite $/ \mu{ }^{37}$. High sequence and structural homology between the two isoform proteins is the accepted reason for this ${ }^{29,38,39}$. This phenomenon can mask the deletion effect on either of the genes on the performance of $\mathrm{mRDT}$ at moderate to high parasite density.

The inclusion of asymptomatic participants adds to the evidence of true pfhrp2/pfhrp3 deletion prevalence. The WHO master protocol for surveillance of pfhrp2/pfhrp3 deletion, which emphasizes a health-center-based approach targeting symptomatic individuals, could underestimate the true magnitude ${ }^{40}$. Detection of the pfhrp2/3 deletions is based on negative PCR results. However, low parasite DNA concentration can lead to a no-amplification result leading to false-negative outcome ${ }^{41,42}$. The use of HRP2-based RDT will likely exert selective pressure on the parasite population and could lead to the spread of pfhrp2/3-deleted parasites $^{43}$.

\section{Conclusion}

This study provides evidence of pfhrp2 and pfhrp3 deletions in $P$. falciparum isolates from Tanzania. This makes it urgent for systematic surveillance of pfhrp $2 / 3$ deletions to understand the prevalence and extent of such deletions in Tanzania. The high 
proportion of pfhrp3 deletion attracts attention and there is now a need to understand what drives these deletions through the transmission season. Findings from this study support the idea of screening for pfhrp2/3 deletions even in mRDT positive samples, bearing in mind the cross-reactivity between the two proteins but also the false positivity of mRDTs due to persistence of plasma HRP2/HRP3 after treatment.

\section{Study limitations}

The study was conducted in the middle of peak transmission season (April - June 2018) and in an area previously reported to have high polyclonal $P$. falciparum infections ${ }^{44,45}$. We might have underestimated gene deletions since we did not estimate the multiplicity of infection in the study areas. The use of a high throughput multiplex qPCR targeting single parasite copies can resolve the veiled effect of polyclonal infection in pfhrp $2 / 3$ gene deletion ${ }^{39}$.

\section{Methods}

\section{Study area and participants}

This study was conducted in Lower-Moshi in Kilimanjaro and Handeni Tanga region (Figure 4). Handeni is in the Tanga region, on the North-Eastern coast of Tanzania. The region is endemic for malaria with a perennial transmission pattern and is known to be a focal area for malaria drug resistance. It has an EIR of about 100 infectious bites per person per year and a perennial prevalence of $25-30 \% 45-47$. The study area has two rainy seasons per year, which denotes the peaks of malaria transmission. The long rainy season is from March-June and the short rainy season is from October-November. The area is located at 309 meters above sea level, $5^{\circ} 22^{\prime} 60^{\prime \prime} \mathrm{N}$ and $38^{\circ} 34^{\prime} 60^{\prime \prime} \mathrm{E}$.

The second study site was Lower Moshi (3021'S, 37020'E), The area is about 800meters above sea level, south of Mount Kilimanjaro. It is one of the sentinel sites for insecticide resistance surveillance under the National Malaria Control Programme. Transmission of malaria in Lower Moshi occurs throughout the year with a prevalence of $<0.1 \% 48$.

\section{Sample size calculation}

The sample size was calculated assuming the following parameters, proportion for pfhrp $2 / 3$ gene deletion is 0.5 (proportion in the population), Power $=0.80$, Alpha $=0.05$ (two-sided), Anticipated difference $=0.1$, Alternative $p=0.4$, Design effect $=2$. The estimated required sample size in each site was $194 * 2=388$ per study site, (Using STATA software). The total estimated minimum sample size was 776 in the two study sites. In this study we enrolled 1013 participants but presented results for only 998 participants, enrolment forms for 15 individuals had missing information and were dropped from the analysis.

\section{Participant recruitment}

Community sensitization meetings were organized, whereby the study personnel explained the study and answered any questions in an open forum before participant recruitment. Enrolment occurred in health facilities in each site, participants were enrolled only after verification of potential eligibility, explaining the study in Swahili (local language) and consent to participate. Each member of the community had an equal chance of being selected as a participant.

\section{Sample collection and Malaria rapid diagnosis}

Whole blood was collected just after the rainy season, from April to June 2018. The diagnosis on enrolled participants was done on-site, whereby a qualitative SD BIOLINE Malaria Ag P.f/Pan test (Standard diagnostics INC. Korea) detects histidine-rich protein II (HRP-II) antigen of Plasmodium falciparum and Plasmodium lactate dehydrogenase (pLDH) for the Plasmodium species.

\section{Blood smear microscopy}

Thick and thin smears were prepared and left to air dry at room temperature, thin smears were carefully sprayed with absolute methanol for fixation. Staining was done with $10 \%$ Giemsa stain for 30 mins, thereafter the stain was washed away by tap water and left to dry. Double reading of the slides was done by a separate microscopist and discordant results were then resolved by a 
third reader and results were presented as the number of parasites in every 200 white blood cells. Microscopists received their training from the Malaria Diagnostic Centre of Excellence in Kisumu Kenya ${ }^{49}$.

\section{DNA Extraction}

Genomic DNA was extracted from dried blood spots (DBS) using a robotic DNA extraction system (Qiasymphony, QIAGEN, Germany) at the London School of Hygiene Tropical Medicine (LSHTM)- UK, the method previously validated and published ${ }^{50,51}$.

PCR confirmation of Plasmodium falciparum DNA

Plasmodium falciparum was detected using a standard nested-PCR technique targeting $18 \mathrm{~S}$ ribosomal RNA, primers and PCR conditions were as previously published ${ }^{52}$.

\section{Estimation of parasite density}

A qPCR that amplifies tRNA methionine of the Plasmodium (PgMET) gene and human beta-tubulin gene (HumTUBB) was used to relatively estimate parasite density in the samples. HumTUBB was used as an internal DNA extraction control and Plasmodium falciparum international standard as a calibrator ${ }^{53}$. Primers and probes used (Table 3), master mixes, and amplification profiles were adopted from Beshir et al, $2013^{54}$.

Table 3

Primers and probes for paraite density qPCR

\begin{tabular}{|llll|}
\hline Primers/Probes & 5' Modification & Sequence & 3' Modification \\
\hline PgMET_F & & 5'-TGAAAGCAGCGTAGCTCAGA & \\
\hline PgMET_R & & 5'-CGCGTGGTTTCGATCCACG & \\
\hline PgMET_pr & FAM & 5'-GGGGCTCATAACCCCCAGGA & BHQ2 \\
\hline HumTuBB_F & & 5'-AAGGAGGTCGATGAGCAGAT & \\
\hline HumTuBB_R & & 5'-GCTGTCTTGACATTGTTGGG & \\
\hline HumTuBB_pr & JOE & 5'-TTAACGTGCAGAACAAGAACAGCAGCT & BHQ2 \\
\hline
\end{tabular}

\section{Pfhrp2 /Pfhrp3 genotyping}

The amplification of pfhrp2/3 genes from DBS samples was done using nested-PCR as described previously ${ }^{55,56}$. Primers used for nest-1 PCR were Pfhrp2-F1 (5'-CAAAAGGACTTAATTTAAATAAGAG-3') and Pfhrp2-R1 (5'-AATAAATTTAATGGCGTAGGCA-3'). Nest-2 primers used were Pfhrp2-F2 (5'-ATTATTACACGAAACTCAAGCAC-3') and Pfhrp2-R1. PCR reaction mix contained a final concentration of $0.2 \mu \mathrm{M}$ for each primer, $0.5 \mu \mathrm{M}$ of deoxynucleoside triphosphate (dNTP) mix (Promega), $0.2 \mathrm{U}$ of Taq-polymerase (AmpliTaq Gold-Applied biosystems), and $5 \mu$ of the DNA template. Amplification thermo-profile for the reaction was $95^{\circ} \mathrm{C}$ for 10 min (Enzyme activation) followed by 40 cycles of $94^{\circ} \mathrm{C}$ for $50 \mathrm{~s}, 50^{\circ} \mathrm{C}$ for $30 \mathrm{~s}$ and $65^{\circ} \mathrm{C}$ for $1 \mathrm{~min}$. Final elongation was done at $70^{\circ} \mathrm{C}$ for $15 \mathrm{~min}$ then at $4^{\circ} \mathrm{C}$ until removed from the machine. Primers used for the amplification of Pfhrp3 gene were Pfhrp3-F1 (5'-AATGCAAAAgGACTTAATTC-3'), Pfhrp3-R1 (5'-TggtgtAagtgatgCGTAGT-3'), Pfhrp3-F2 (5'-

AAATAAGAGATTATTACACGAAAG-3') and Pfhrp3-R1. Master mix and amplification profiles were the same as for Pfhrp2.

\section{Data Analysis}

A descriptive analysis was performed using Stata 16 software (StataCorp LLC, TX, USA), with proportions and frequencies detailed in tables and figures. A log transformation of relative parasitaemia was applied to compare the mean and median values. Box plots were used to show the median, the 25th, and 75th percentiles of the non-parametric test.

\section{Ethics approval}

This study was assessed and approved by the Kilimanjaro Christian Medical University College Research Ethics Review Committee and given ethical clearance certificate \# 2238 of the research proposal \# 1084. Consent from study participants was 
sought before enrolment, guidelines and regulations that safeguard participants were also observed.

\section{Declarations}

\section{Acknowledgments}

We thank the study participants from Handeni and Moshi for their involvement in this study. This could have not been possible without the support of the district medical officers from both sites. This study was funded through the DELTAS Africa Initiative grant \# DEL-15-011 to THRiVE-2. The DELTAS Africa Initiative is an independent funding scheme of the African Academy of Sciences (AAS), Alliance for Accelerating Excellence in Science in Africa (AESA) and supported by the New Partnership for Africa's Development Planning and Coordinating Agency (NEPAD Agency) with funding from the Wellcome Trust grant \# 107742/Z/15/Z and the UK government.

\section{Author contributions}

RDK, RAK, CD, FWM and KBB wrote and review the main study proposal and experimental design of the study. RDK, FFT and KBB performed data analysis. RDK and KBB performed the molecular laboratory analysis. RDK wrote the manuscript, which was revised by and RAK, JJM, FFT, CJS, KBB. All authors read and approved the final manuscript.

\section{Competing interests}

The authors declare no competing interests.

\section{References}

\section{Figures}

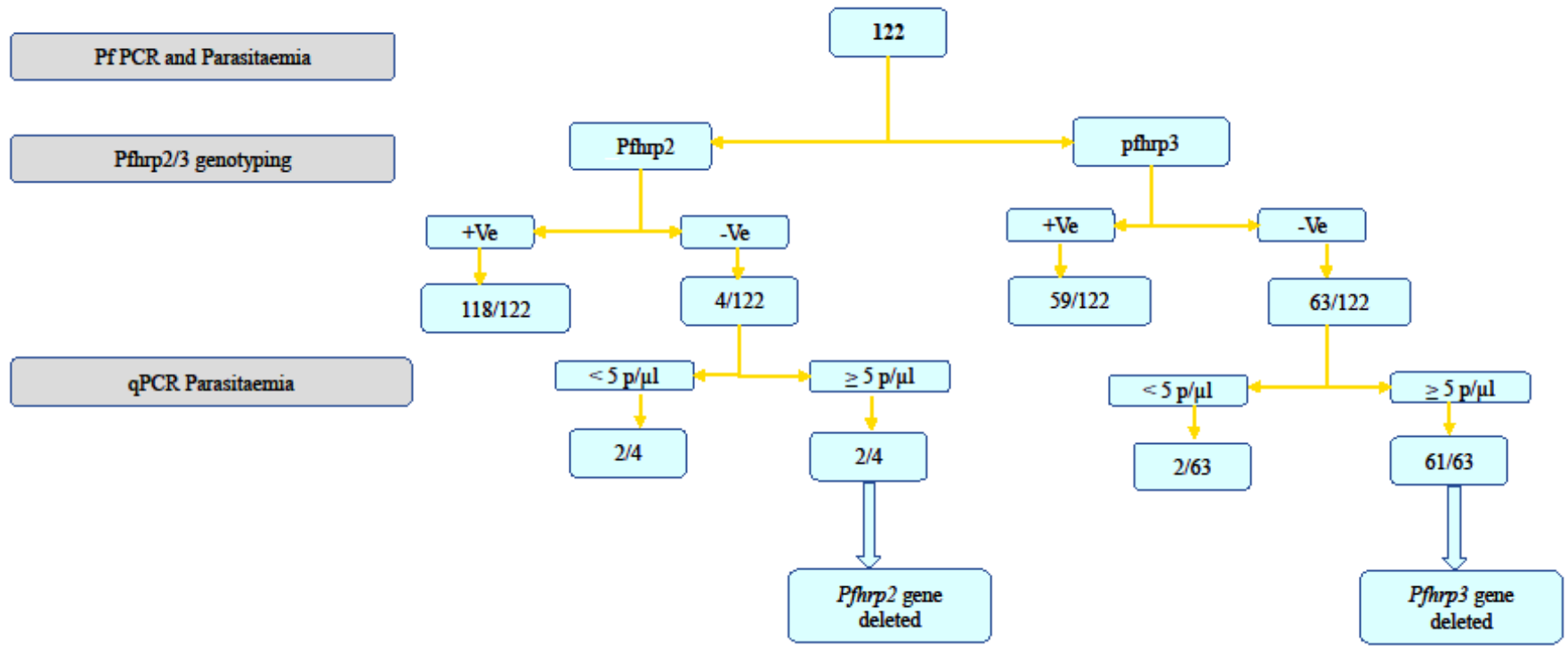

Figure 1 


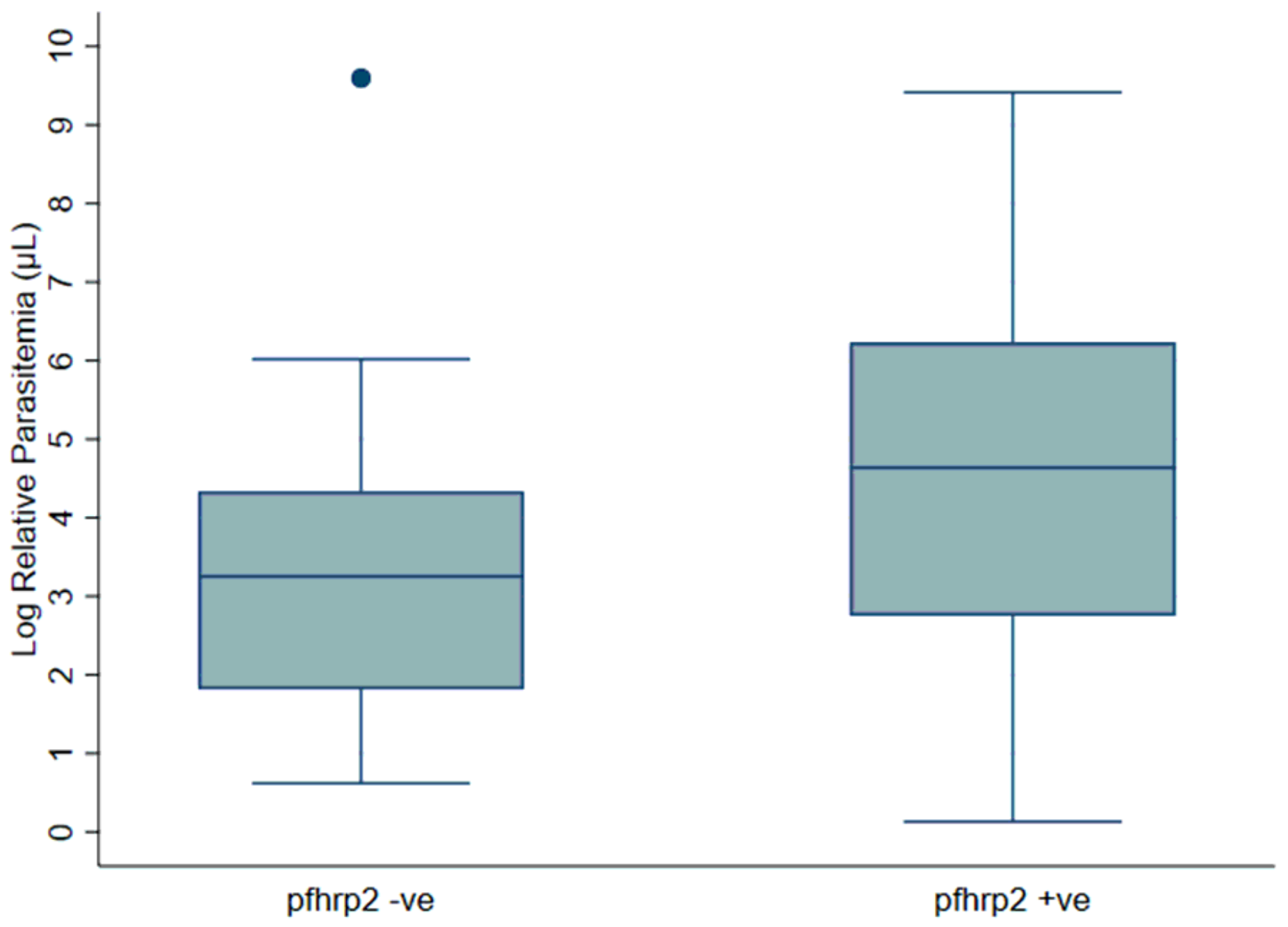

Figure 2

Parasitemia levels for pfhrp-2 gene negative and positive 


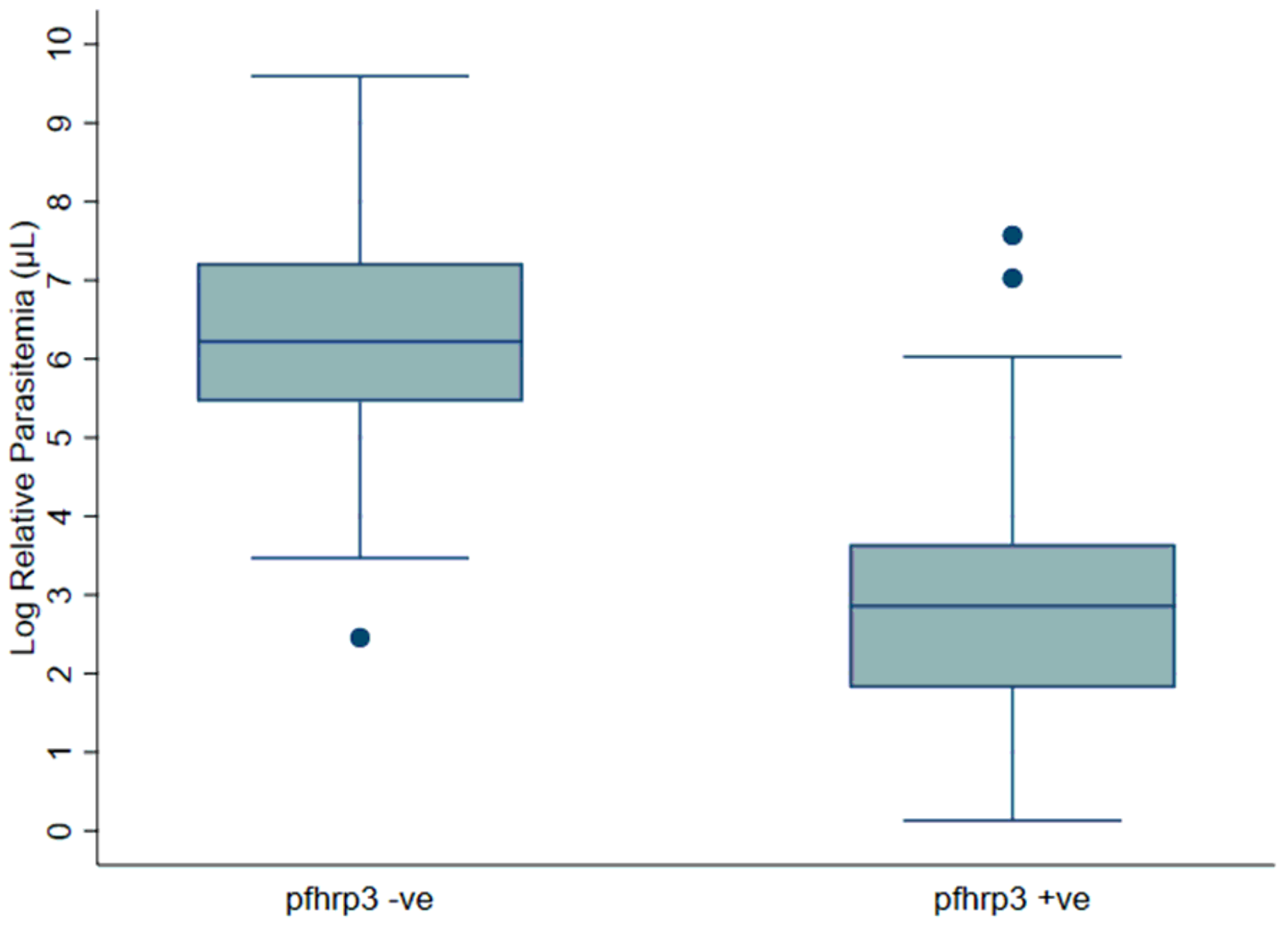

Figure 3

Parasitemia levels for pfhrp-3 gene negative and positive 


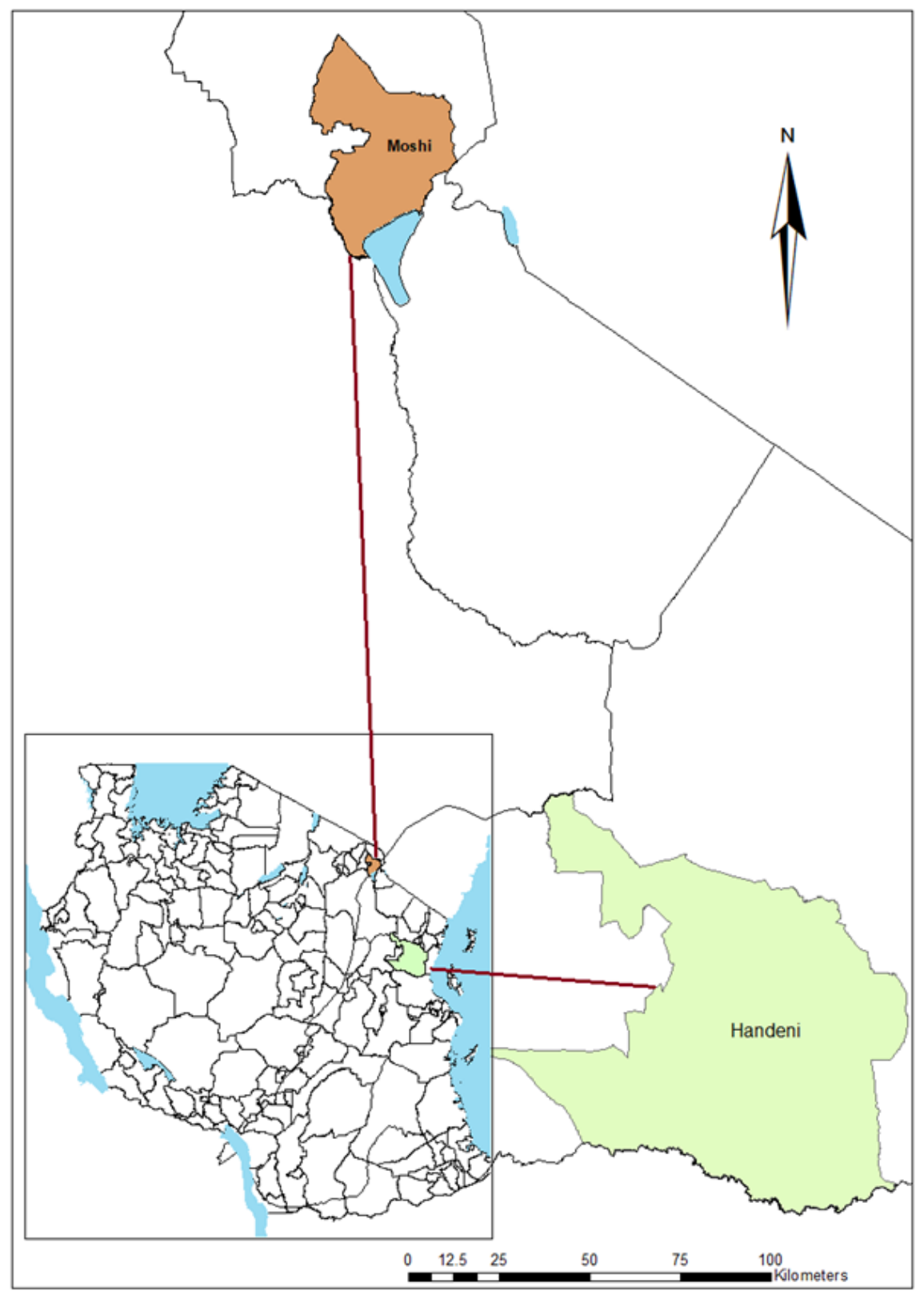

Figure 4

A map of Tanzania showing the study areas (map created using ArcGIS software v10.3) 